Annuaire suisse de politique de développement

22-1 | 2003

Faits et statistiques 2003

\title{
1. Politique extérieure
}

\section{Christoph Stamm}

\section{(2) OpenEdition \\ Journals}

Édition électronique

URL : http://journals.openedition.org/aspd/595

DOI : 10.4000/aspd.595

ISSN : 1663-9669

\section{Éditeur}

Institut de hautes études internationales et du développement

\section{Édition imprimée}

Date de publication : 1 mars 2003

Pagination : 3-11

ISBN : 2-88247-049-5

ISSN : $1660-5934$

Référence électronique

Christoph Stamm, «1. Politique extérieure », Annuaire suisse de politique de développement [En ligne] 22-1 | 2003, mis en ligne le 23 mars 2010, consulté le 08 septembre 2020. URL : http:// journals.openedition.org/aspd/595; DOI : https://doi.org/10.4000/aspd.595 


\section{POLITIQUE EXTÉRIEURE*}

E N 2002, la politique extérieure de la Suisse a été marquée par deux grands événements. D’une part, la Suisse est devenue, en septembre 2002, membre à part entière de l'Organisation des Nations unies, après que le peuple eut approuvé ce projet lors d'une votation au printemps. D'autre part, les relations de la Suisse avec l'Union européenne (UE) ont acquis de nouvelles bases avec l'entrée en vigueur, en juin 2002, des accords bilatéraux. Par ailleurs, le lancement de nouvelles négociations avec l'Union européenne (bilatérales II) confirme la volonté de poursuivre la voie bilatérale.

Ces deux événements d'envergure achèvent une décennie plutôt mouvementée sur le plan de la politique extérieure, qui a débuté en 1992 avec le rejet de l'EEE par le peuple et avec l'adhésion de la Suisse au groupe de la Banque mondiale et du Fonds monétaire international. La politique de sécurité et de paix illustre également la réorientation de la politique extérieure de la Suisse. Pour la première fois, des soldats suisses armés ont participé à une mission de paix (KFOR, Force de paix au Kosovo) et la promotion civile de la paix se fonde désormais sur une loi fédérale.

\subsection{LA SUISSE ET L'ONU}

\section{$\square$ Votation sur l'adhésion à l'ONU}

Le 3 mars 2002, le peuple suisse a adopté par 54,6\% des voix l'initiative populaire pour l'adhésion de la Suisse à l'Organisation des Nations unies (ONU). Lors de ce scrutin, la participation a atteint $58 \%$ et 12 cantons se sont prononcés en faveur de l'initiative, tandis que 11 l'ont rejetée ${ }^{1}$. C'est dire que l'initiative l'a remporté de justesse au niveau cantonal.

Si l'on compare ce résultat à celui du vote de 1986 sur le référendum contre l'adhésion à l'ONU - la majorité du peuple et tous les cantons avaient alors refusé l'adhésion -, la part des oui s'est accrue de plus de $20 \%$. L'écart qui sépare les résultats de ces deux scrutins illustre le revirement de l'opinion publique suisse ces seize dernières années, notamment depuis la fin de la guerre froide.

Dès lors qu'il avait l'appui du peuple et des cantons, plus rien n'empêchait le Conseil fédéral de transmettre la demande d'adhésion de la Confédération helvétique à l'Organisation des Nations unies. Ce fut chose faite le 20 juin $2002^{2}$.

데 ASTM 2002, pp. 207-209.

* Par Christoph Stamm, politologue.

1 Confédération suisse, Initiative populaire fédérale "pour l'adhésion de la Suisse à l'Organisation des Nations unies $(O N U) »$, votation $n^{\circ} 485,<w w w . a d m i n . c h>$.

2 Conseil fédéral, Demande d'adhésion de la Suisse à l'ONU incluant une déclaration concernant la neutralité, juin 2002, <www.uno.admin.ch>. 


\section{$\square$ La politique de la Suisse au sein de l'ONU}

Avant l'adhésion officielle de la Suisse à l'ONU, le Conseil fédéral a formulé en mai 2002 la politique qu'il entend mener au sein de cette organisation et fixé ses priorités pour la $57^{\mathrm{e}}$ Assemblée générale de $1^{\prime} \mathrm{ONU}{ }^{3}$.

Désormais membre à part entière de l'ONU, la Suisse possède le droit de vote à l'Assemblée générale et est également éligible. Elle peut donc être élue au sein du Conseil économique et social (ECOSOC), de ses commissions et du Conseil de sécurité de l'ONU. C'est pourquoi le Conseil fédéral entend prévoir un programme de candidatures qui devrait permettre à la Suisse d'être représentée au sein des organes internationaux tout en mettant en valeur des personnalités suisses. Il met ici la priorité sur la nomination de la Suisse au sein de l'ECOSOC et de la Commission des droits de l'homme. Pour y parvenir, la Suisse devra toutefois se soumettre à des systèmes de rotation parfois relativement longs.

La Suisse peut par ailleurs faire valoir ses grandes préoccupations en intervenant dans les débats de l'Assemblée plénière ou dans le cadre de sous-commissions, en proposant ou en cosignant des résolutions et en participant aux consultations informelles qui accompagnent la préparation des résolutions et des décisions ${ }^{4}$.

L'attitude de la suisse au sein de l'ONU découle des objectifs définis dans le Rapport sur la politique extérieure 2000, notamment sur les priorités qu'il fixe en matière de politique extérieure: maintien et promotion de la sécurité et de la paix; accroissement de la prospérité commune et promotion de la cohésion sociale; engagement en faveur des droits de l'homme, de la démocratie et des principes de l'Etat de droit; préservation du milieu naturel. La consolidation du rôle du siège genevois de l'ONU constitue également une priorité pour la Suisse ${ }^{5}$.

Une vingtaine d'organisations non gouvernementales (ONG) suisses ont formé une plate-forme afin de prendre part pleinement aux activités de la Suisse au sein de l'ONU6'. Elles ont adressé leurs revendications au Conseil fédéral en août 2002 dans un premier document, dans lequel elles mettent le doigt sur les lacunes de la politique d'information de la Confédération et sur les problèmes de cohérence qui vont immanquablement se poser entre les différents domaines de la politique. Les ONG déclarent qu'il importe de placer les droits fondamentaux et les droits de l'homme, les normes sociales et les objectifs du développement durable au tout premier plan. Elles relèvent également que la Suisse n'a ratifié ni la Convention alpine ni le Protocole de Kyoto de la Convention sur le climat.

Dans la perspective de la $57^{\mathrm{e}}$ session de l'Assemblée générale, les ONG ont mis l'accent sur deux objectifs qu'elles jugent prioritaires: d'une part, la Cour pénale internationale doit devenir opérationnelle dès que possible et, d'autre part, la Suisse doit reconnaître l'autorité suprême de l'ONU telle qu'elle est inscrite dans la Charte. Cette reconnaissance implique en particulier que la Suisse s'engage à

3 Confédération, Le Conseil fédéral définit la politique qu'il entend mener à l'ONU et fixe ses priorités pour la $57^{2}$ session de l'Assemblée générale des Nations unies, communiqué de presse, 29.5.02.

4 Les résolutions sont des recommandations et n'ont de ce fait aucun caractère juridique contraignant. Les décisions règlent les questions de procédure.

5 DFAE, 57e Assemblée générale de l'ONU. Première participation de la Suisse en qualité de membre, 2002, p. 11.

6 Plattform der Schweizerischen NGO zuhanden des Bundesrates anlässlich der ersten UNO-Generalversammlung mit Schweizer Vollmitgliedschaft, 26.8.02. 
n'approuver que des opérations de maintien de la paix décidées par l'ONU ou par l'OSCE et, le cas échéant, à y prendre part dans la mesure de ses moyens.

\section{$\square$ L'adhésion de la Suisse à l'ONU}

La Suisse a été admise au sein de l'ONU le 10 septembre 2002, à l'occasion de l'ouverture de l'Assemblée générale annuelle de l'ONU, sur la base d'une recommandation du 24 juillet 2002 du Conseil de sécuritép. En présentant la résolution d'admission de la Suisse au nom des Etats voisins, le ministre des Affaires étrangères de la France a relevé que la Suisse était le seul pays dont l'adhésion à l'ONU ait été soumise au scrutin populaire et que cette consultation du peuple pourrait contribuer à renforcer l'orientation démocratique de l'organisation.

Dans son discours devant l'Assemblée générale, le président de la Confédération, Kaspar Villiger, a exprimé la volonté de la Suisse de travailler au sein de l'ONU dans un esprit à la fois constructif et critique. Il a également rappelé quelques particularités de l'identité politique suisse et souligné enfin que la Suisse resterait un pays neutre ${ }^{8}$.

Avec l'adhésion de la Suisse et du Timor-Oriental (25 septembre 2002), le nombre des Etats membres de l'ONU est passé à 191. Ainsi, tous les Etats reconnus par la communauté internationale, à l'exception du Vatican, sont désormais membres de l'Organisation des Nations unies.

\section{$\square$ Premières activités lors de l'Assemblée générale de l'ONU}

Le jour de l'adhésion de la Suisse à l'ONU, l'Assemblée générale de l'organisation ouvrait sa $57^{e}$ session'. L'Assemblée générale est l'un des principaux organes de l'ONU. Elle possède une compétence générale pour aborder et traiter tous les questions et problèmes qui relèvent de la Charte de l'ONU. L'assemblée se réunit une fois par année et assume des fonctions non seulement politiques mais aussi organisationnelles ${ }^{10}$. La session annuelle ordinaire débute toujours le deuxième mardi de septembre et s'ouvre sur un débat général de deux semaines. Les travaux se poursuivent ensuite au sein des six commissions ou en plénum jusqu'au début de l'année suivante.

En 2002, le débat général a notamment été consacré à un programme de réforme proposé par le secrétaire général, à une réforme du Conseil de sécurité, à la place à réserver au multilatéralisme et à une amélioration de la coopération entre les acteurs de l'aide humanitaire. L'Assemblée générale devait par ailleurs élire cinq nouveaux membres non permanents au Conseil de sécurité ${ }^{11}$. La Suisse a pris part aussi bien au débat qu'aux votes. Elle a salué le lancement du programme de réforme qui vise avant tout à renforcer les capacités de l'ONU dans la protection et dans la promotion des droits de l'homme, à rationaliser et à

«Grosser Tag für die Schweiz in New York», Neue Zürcher Zeitung, 11.9.02.

DFAE, Discours de M. Kaspar Villiger, président de la Confédération, à l'occasion de l'adhésion de la Suisse à l'ONU, 10.9.02.

9 DFAE, La Suisse à l'ONU: bilan intermédiaire. Avancement des travaux de l'Assemblée générale, octobre 2002, <www.uno.admin.ch>.

10 Nations unies, A propos de l'Assemblée générale, <www.un.org/french/ga/57/about.htm>.

11 Voici les cinq pays qui ont été nommés pour deux ans: Allemagne, Espagne, Chili, Angola et Pakistan. 
améliorer la communication, ainsi qu'à réduire le nombre des séances et des rapports. Pour ce qui est des élections et des candidatures, l'adhésion de la Suisse à l'ONU a déjà porté ses fruits: le professeur Walter Kälin a été élu au Comité des droits de l'homme de l'ONU; le diplomate Valentin Zellweger est devenu vice-président de la $6^{\mathrm{e}}$ Commission de l'Assemblée générale; et, enfin, l'ambassadeur Jean-Marc Boulgaris a été élu à la vice-présidence du Comité exécutif du Haut Commissariat des Nations unies pour les réfugiés ${ }^{12}$.

Dans le cadre des six commissions spécialisées de l'Assemblée générale, au sein desquelles les divers projets de résolution sont débattus et adoptés, la Suisse peut prendre position sur des points concrets et faire valoir son avis sur la politique mondiale et sur la politique extérieure.

- Première commission: Désarmement et sécurité internationale. La Suisse entend favoriser l'élimination des armes de destruction massive et une plus grande transparence en matière de désarmement. Dans le domaine de la sécurité humaine, elle souhaite promouvoir une initiative lancée conjointement avec la France visant à définir des mécanismes de traçage des armes légères et de petit calibre.

- Deuxième commission: Questions économiques et financières, développement. La Confédération appuie la nouvelle stratégie pour le développement de l'Afrique $(\mathrm{NEPAD})^{13}$. Elle s'engage à améliorer la coopération entre les divers acteurs du développement (gouvernements, organisations internationales, société civile et secteur privé) et appelle à une application plus rigoureuse des conventions conclues dans le domaine de la protection de l'environnement.

- Troisième commission: Questions sociales, humanitaires et culturelles. Les diplomates suisses accordent une place particulière à l'adoption par l'Assemblée générale d'un protocole additionnel contre la torture.

- Quatrième commission: Politiques spéciales et décolonisation. Cette commission s'occupe essentiellement du maintien de la paix (amélioration des missions de l'ONU) et des questions politiques spéciales, telles que la situation au Proche-Orient. Dans ce cadre, la Suisse demande le respect des Conventions de Genève dans les territoires occupés.

- Cinquième commission: Questions administratives et budgétaires. La Suisse s'est prononcée en faveur d'une croissance nulle du budget ordinaire. Elle souhaite toutefois que l'on consacre davantage de ressources à la défense des droits de l'homme et à la protection de l'environnement. Une plus grande transparence devrait par ailleurs être de mise dans le poste des mesures de maintien de la paix, qui engloutit deux tiers de toutes les dépenses.

- Sixième commission: Questions juridiques. La Confédération met ici l'accent sur le débat concernant la Cour pénale internationale ainsi que sur les Conventions de Genève et sur leurs protocoles additionnels.

Pendant sa première année de participation aux travaux de l'ONU, la Suisse devra prendre position sur quelque 300 résolutions. Bénéficiant de ressources

12 Confédération, Election du professeur Walter Kälin au Comité des droits de l'homme de l'ONU, communiqué de presse, 9.9.02.; idem, Un diplomate suisse est élu vice-président de la Commission de l'ONU qui s'occupe du droit international, communiqué de presse, 24.9.02.

13 Voir l'Annuaire Suisse-Tiers Monde 2002, p. 205. 
limitées et de structures qui doivent être confirmées, les diplomates suisses dont s'est entouré l'ambassadeur Jenö Staehelin devront donc s'efforcer de traiter rapidement les diverses propositions de résolution.

\subsection{LOI FÉDÉRALE SUR DES MESURES DE PROMOTION CIVILE DE LA PAIX ET DE RENFORCEMENT DES DROITS DE L'HOMME}

Sur la base d'un arrêté fédéral de juin 2000, le Département fédéral des affaires étrangères (DFAE) a été chargé d'élaborer un projet de loi fédérale sur des mesures de promotion civile de la paix et de renforcement des droits de l'homme. Le 23 octobre 2002, le Conseil fédéral a soumis au Parlement le projet de cette nouvelle loi et le message correspondant ${ }^{14}$.

L'adoption d'une loi était devenue nécessaire, car la Confédération alloue dans ce domaine des moyens de près de 40 millions de francs sans que ces dépenses se fondent sur une base législative formelle. La nouvelle loi fédérale présente avant tout un caractère technique et ne comprend aucune innovation en matière d'orientation politique ou opérationnelle ${ }^{15}$. Les politiques et les programmes seront à l'avenir définis dans le cadre des demandes de crédits-cadres qui seront présentées conformément à la loi.

C'est pourquoi le Conseil fédéral a non seulement soumis aux Chambres fédérales le projet de loi, mais aussi un message concernant l'ouverture d'un créditcadre pour des mesures de gestion civile des conflits et de promotion des droits de l'homme. Ce message prévoit un crédit global de 240 millions de francs qui permettra au DFAE d'optimiser ses activités dans les deux domaines concernés en les planifiant à plus long terme. Il est prévu que le budget annuel augmentera progressivement pour passer de 44,5 millions en 2003 à 62,5 millions en $2007^{16}$. Le message concernant l'ouverture d'un crédit-cadre commence par une analyse et une rétrospective des efforts consentis jusqu'alors, puis il définit les buts, les principes, les domaines d'actions ainsi que les priorités thématiques de la politique suisse dans ce secteur.

Annuaire 2003, n ${ }^{\circ}$, chap. 11, «Politique en matière de sécurité et politique de paix».

La promotion des droits de l'homme comprend ainsi les deux domaines d'action que sont les initiatives diplomatiques et les dialogues sur les droits de l'homme. La gestion civile des conflits prévoit les bons offices et la médiation, les programmes de gestion civile des conflits, un pool d'experts pour la promotion civile de la paix, des initiatives diplomatiques thématiques et des partenariats

14 L'entrée en vigueur de la nouvelle loi est prévue pour janvier 2004. Confédération, Promotion civile de la paix et renforcement des droits de l'homme: nouvelle loi fédérale et crédit-cadre, communiqué de presse, 23.10.02; Conseil fédéral, Message concernant la loi fédérale sur des mesures de promotion civile de la paix et de renforcement des droits de l'homme du 23 octobre 2002, Feuille fédérale (02.077), 2002, pp. 7063-7073.

15 Pour ce qui est des priorités et des instruments de la promotion de la paix, voir DFAE, Stratégies d'action en faveur de la paix. Législature 2000-2003, décembre 1999; idem, Prévenir la violence. Participer à l'instauration de la paix. Consolider la démocratie. La politique de paix de la Direction politique du DFAE, juillet 2001. Pour ce qui est de la politique suisse des droits de l'homme, voir Conseil fédéral, Rapport sur la politique suisse des droits de l'homme, février 2000.

16 «Mehr Mittel für zivile Friedensförderung», Neue Zürcher Zeitung, 24.10.02. 
(pour le développement de stratégies de politique de paix par exemple). Les questions de droit constitutionnel, décentralisation et partage du pouvoir, les médias et les conflits armés ainsi que la sécurité humaine sont considérés comme thèmes prioritaires.

Le 23 octobre 2002, le Conseil fédéral a également présenté son Rapport sur les possibilités et les limites de l'affectation de volontaires à l'étranger dans le cadre de la promotion civile de la paix ${ }^{17}$. Ce rapport accorde une place particulière au pool d'experts pour la promotion civile de la paix (PEP) créé en 2000. Les envois de ces «experts de la paix » à l'étranger se sont révélés positifs, mais le Conseil fédéral n'en poursuivra pas moins ses efforts pour améliorer encore la préparation, le suivi et l'évaluation de leurs missions.

\subsection{CRÉATION D'UNE COMMISSION FÉDÉRALE DES DROITS DE L'HOMME}

En 1993 déjà, la Conférence mondiale sur les droits de l'homme a souligné, dans son plan d'action auquel la Suisse a pleinement souscrit, qu'il importait de créer des commissions nationales des droits de l'homme. Ce n'est toutefois que depuis février 2000, date à laquelle le Conseil fédéral a publié un rapport sur la politique suisse des droits de l'homme, que la création d'une commission fédérale des droits de l'homme est à l'ordre du jour. Une centaine d'ONG revendiquent la mise en place d'une instance indépendante chargée de veiller au respect des droits de $1^{\prime}$ homme en Suisse ${ }^{18}$. En décembre 2001, une initiative parlementaire a été déposée tant au Conseil national qu'au Conseil des Etats pour demander la création d'une telle commission. En septembre 2002, la Commission de politique extérieure du Conseil des Etats a rejeté cette initiative pour charger le Conseil fédéral d'établir un rapport sur la possibilité de créer une commission des droits de l'homme $^{19}$. La Commission des institutions politiques du Conseil national a pour sa part recommandé l'adoption de l'initiative. Etant donné que 109 des 200 parlementaires ont signé cette initiative, une majorité de la Chambre basse devrait l'approuver ${ }^{20}$.

ASTM 2001, Rapport sur la politique suisse des droits de l'homme (pp. 248-250).

La Suisse possède déjà divers instruments ${ }^{21}$ permettant de contrôler le respect de certains droits de l'homme (lutte contre le racisme, égalité entre hommes et femmes), mais aucune commission chargée de veiller au respect des différents droits économiques, sociaux, culturels, politiques et civiques. Selon un rapport diffusé par les auteurs de l'initiative, une commission des droits de l'homme pourrait assumer les tâches suivantes: veiller à l'application en Suisse de conventions internationales sur les droits de l'homme; traiter les violations des

17 Confédération, Rapport du Conseil fédéral sur les possibilités et les limites de l'affectation de volontaires à l'étranger dans le cadre de la promotion civile de la paix, communiqué de presse, 23.10.02.

18 Groupe de travail Commission pour les droits humains, Commission fédérale pour les droits humains, septembre 2002.

19 Parlement fédéral, Conseil des Etats, Commission fédérale des droits de l'homme, postulat 02.3394 de la Commission de politique extérieure CE, 9.9.02.

20 «Die Menschenrechte im eigenen Land durchsetzen», Tagesanzeiger, 4.10.02.

21 Il s'agit de la Commission fédérale contre le racisme, du Service de lutte contre le racisme et du Bureau fédéral de l'égalité entre femmes et hommes, tous trois intégrés au Département fédéral de l'intérieur. 
droits de l'homme sur les plans juridique et politique; créer des procédures de conciliation; conseiller le Parlement et le gouvernement en matière de droits de l'homme; sensibiliser l'opinion publique aux questions relevant des droits de l'homme et contribuer à consolider et à développer les instruments nationaux et internationaux de protection des droits de l'homme ${ }^{22}$.

Relevons que la politique des droits de l'homme concerne les politiques aussi bien intérieure qu'extérieure. Une commission fédérale des droits de l'homme qui étendrait également son travail d'observation et ses conseils à la politique extérieure permettrait donc d'améliorer la cohérence entre les politiques intérieure et extérieure dans ce domaine particulier.

\section{4. « STRATÉGIE DE POLITIQUE EXTÉRIEURE DE LA SUISSE POUR L'EUROPE DU SUD-EST »}

Le 24 avril 2002, le conseil fédéral a adopté23 une Stratégie pour l'Europe du Sud-Est ${ }^{24}$. Après celle pour le bassin sud et est de la Méditerranée, adoptée le 11 avril 2001, c'est la deuxième stratégie de ce type, qui tient compte des priorités géographiques de la politique extérieure telles qu'elles sont définies dans le Rapport sur la politique extérieure 2000.

La stabilité de cette région joue un tel rôle pour l'Europe, et la guerre et les crises qui l'ont secouée ces dix dernières années ont eu de telles conséquences que la Suisse a décidé d'engager des moyens importants à différents niveaux pour contribuer à y faire régner la paix. La stratégie identifie les intérêts de la Suisse, fixe les objectifs de sa politique à moyen terme (trois à cinq ans) et rappelle les instruments dont elle dispose pour les atteindre. Les principaux intérêts de la Suisse dans la région sont les suivants: prévention de nouveaux conflits armés; développement de l'Europe du Sud-Est en tant que partenaire politique et économique à part entière; prévention de flux massifs de réfugiés et de migrations; lutte contre l'extrémisme politique et la criminalité organisée; intégration dans les structures euro-atlantiques. Cette stratégie de politique extérieure a pour principal objectif d'améliorer la coordination des activités suisses en Europe du Sud-Est ${ }^{25}$. Le texte affirme que la Suisse est considérée comme un partenaire important dans la région. A ce titre, elle assumera sa part de la coopération internationale tant que la situation l'exige et elle est décidée à débloquer les moyens nécessaires pour poursuivre les activités qu'elle juge prioritaires.

22 Groupe de travail Commission pour les droits humains, Commission fédérale pour les droits humains, septembre 2002.

23 Conseil fédéral, Stratégie de politique extérieure de la Suisse pour l'Europe du Sud-Est, avril 2002.

24 La région appelée Europe du Sud-Est regroupe les pays suivants : Albanie, Bosnie-Herzégovine, Bulgarie, Croatie, Macédoine, Moldavie, République fédérale de Yougoslavie et Roumanie.

25 Voici les départements et offices fédéraux qui mènent des activités dans la région: Département fédéral des affaires étrangères, Direction du développement et de la coopération, Secrétariat d'Etat à l'économie, Office fédéral des réfugiés, Département fédéral de la défense, de la protection de la population et des sports. La coordination des activités incombe au DFAE. 


\subsection{POLITIQUE EXTÉRIEURE EN MOUVEMENT}

Un ouvrage paru en allemand ${ }^{26}$ analyse l'évolution de la politique extérieure de la Suisse au cours des dix dernières années. Les auteurs s'interrogent d'une part sur l'influence que les changements mondiaux exercent sur cette politique et traitent d'autre part des processus de décision et de l'influence des facteurs intérieurs sur la politique extérieure. L'ouvrage se fonde sur les travaux d'un programme national de recherche achevé en 2000: Fondements et possibilités de la politique extérieure suisse (PNR 42) ${ }^{27}$.

Selon les auteurs, l'évolution du contexte international s'est sensiblement accélérée depuis la fin de la guerre froide et elle a eu deux effets sur la politique extérieure de la Suisse: «D'une part, le multilatéralisme a gagné en importance et, d'autre part, l'écart entre politique intérieure et politique extérieure s'est rétréci. La mise en place d'une politique extérieure cohérente doit donc faire face à un nombre plus grand de défis et de conflits d'intérêts. $»^{28}$ La nouvelle orientation de la politique extérieure (que l'on observe également dans d'autres pays) se caractérise par les éléments suivants: acteurs toujours plus nombreux, prépondérance de questions relevant de la politique extérieure qui touchent aussi bien l'individu que l'ensemble de la société, plus grande transparence et, en raison des sujets traités, nouvelle obligation de rendre des comptes. En Suisse, ces caractéristiques sont encore accentuées puisque la population bénéficie d'un droit de consultation en matière de politique extérieure (référendum en matière de traités internationaux). De plus, les droits démocratiques (référendum et initiative populaire) conçus pour les questions de politique intérieure sont de plus en plus utilisés pour des sujets qui relèvent de la politique extérieure.

Si l'évolution actuelle se poursuit, les auteurs pensent que «le fait de désigner la politique extérieure de politique intérieure mondiale ne reflètera plus simplement un jeu de l'esprit, mais une réalité $»^{29}$.

26 Goetschel Laurent, Bernath Magdalena, Schwarz Daniel, Schweizerische Aussenpolitik. Grundlagen und Möglichkeiten, Berne: Editions NZZ, 2002.

27 Direction du programme: Laurent Goetschel. Voir le site du PNR 42: <www.snf.ch/NFP/nfp42/ home.htm>.

28 Goetschel Laurent, Bernath Magdalena, Schwarz Daniel, Schweizerische Aussenpolitik. Grundlagen und Möglichkeiten, op. cit., p. 222.

29 Ibid., p. 228. 


\section{SOURCES}

Goetschel Laurent, Politique extérieure en mouvement, synthèse du PNR 42, octobre 2000.

Goetschel Laurent, Bernath Magdalena, Schwarz Daniel, Schweizerische Aussenpolitik. Grundlagen und Möglichkeiten, Berne: Editions NZZ, 2002.

Confédération, Arrêté fédéral sur l'adhésion de la Suisse à l'Organisation des Nations unies du 14 décembre 1984, votation $\mathrm{n}^{\circ} 338,1986,<w w w . a d m i n . c h / c h / f / p o r e / v a>$.

Confédération, Initiative populaire "pour l'adhésion de la Suisse à l'Organisation des Nations unies $(O N U) »$, votation n ${ }^{\circ} 485,2002,<w w w . a d m i n . c h / c h / f / p o r e / v a>$.

Conseil fédéral, Message concernant la loi fédérale sur des mesures de promotion civile de la paix et de renforcement des droits de l'homme du 23 octobre 2002, Feuille fédérale (02.077), 2002, pp. $7063-$ 7073.

Conseil fédéral, Rapport sur la politique suisse des droits de l'homme, février 2000.

Conseil fédéral, Stratégie de politique extérieure de la Suisse pour l'Europe du Sud-Est, avril 2002.

DFAE, $57^{e}$ Assemblée générale de l'ONU. Première participation de la Suisse en qualité de membre, 2002.

DFAE, Droits de l'homme, 2002.

DFAE, La Suisse à l'ONU : bilan intermédiaire. Avancement des travaux de l'Assemblée générale, 2002.

DFAE, La Suisse et le monde, revue du Département fédéral des affaires étrangères, éditions 2/2002 et 4/2002.

DFAE, Prévenir la violence. Participer à l'instauration de la paix. Consolider la démocratie. La politique de paix de la Direction politique du DFAE, juillet 2001.

DFAE, Stratégie d'action en faveur de la paix. Législature 2000-2003, décembre 1999.

DFAE, Promotion civile de la paix et renforcement des droits de l'homme : nouvelle loi fédérale et créditcadre, 23.10.02.

Groupe de travail Commission pour les droits humains, Commission fédérale pour les droits humains, septembre 2002.septembre 2002.

Menschenrechte Schweiz, humanrights.ch, $\mathrm{n}^{\mathrm{o}} 3$, septembre 2002,

Plattform der Schweizerischen NGO zuhanden des Bundesrates anlässlich der ersten UNO-Generalversammlung mit Schweizer Vollmitgliedschaft, Berne, août 2002.

Neue Zürcher Zeitung, 11.9.02, 24.10.02.

Tagesanzeiger, 4.10.02.

\section{SITES INTERNET}

Organisation des Nations unies : <www.un.org $>$ et $<$ www.un.org/french $>$.

ONU, Assemblée générale, 57e session, $2002:<w w w . u n . o r g / f r e n c h / g a / 57 / i n d e x . h t m l>$.

DFAE, informations sur l'ONU : <www.uno.admin.ch $>$.

Mission de la Suisse auprès de l'ONU à Genève : <www.eda.admin.ch.geneva_miss/e/home> .

Mission de la Suisse auprès de l'ONU à New York : <www.eda.admin.ch.newyork_miss/e/home>.

Menschenrechte Schweiz MERS : <www.humanrights.ch>. 